\title{
Perceção de Competências de Aprendizagem em Ambientes Online no Ensino da Educação Física
}

\section{Understanding Learning Skills in Online Learning Environments in Physical Education Teaching}

\begin{abstract}
Resumo: Poderão os ambientes online favorecer as competências de aprendizagem, tal como os estudantes do ensino fundamental as percebem? Neste artigo analisamos o impacto de novos cenários pedagógicos online na perceção das competências de aprendizagem na disciplina de Educação Física do ensino fundamental. Participaram na investigação 66 alunos do $6 .^{\circ}$ ano de escolaridade, de uma escola do distrito do Porto- Portugal. No final do ano letivo, os estudantes responderam a uma escala de competências de autoaprendizagem, adaptada para a aprendizagem em ambientes online. Os resultados do estudo mostram que os ambientes virtuais de aprendizagem, ancorados num design centrado no desenvolvimento de competências e num modelo pedagógico baseado nos princípios do construtivismo, da autonomia e da interação podem ter efeitos muito positivos na perceção das competências de aprendizagem nas dimensões consideradas: Aprendizagem Ativa ou Aceitação da Responsabilidade Pessoal pela Aprendizagem, Iniciativa de Aprendizagem e Orientação para a Experiência e Autonomia na Aprendizagem.

Palavras-chave: competências de aprendizagem. Perceção de aprendizagem. Ambientes online.
\end{abstract}

\begin{abstract}
Can online environments promote learning skills such that basic education students understand them? This paper examines the impact of new online educational scenarios as to how self-learning skills are perceived. The research covered 66 students, $6^{\text {th }}$ year, of one school in the district of Porto, Portugal. At the end of the academic year, students responded to a range of self-learning skills adapted to learning in online environments. The results of the study show that virtual learning environments, anchored in a design focused on the development of skills and in a teaching model based on the principles of constructivism, autonomy and interaction can be positive in how basic education students perceive learning skills, according to the following dimensions: Active Learning or Accepting Personal Responsibility through Learning, Learning Initiative and Guidance on Learning Experience and Autonomy.

Keywords: learning skills. Perception learning. Online environments.
\end{abstract}

RIBEIRO, Maria da Glória Oliveira Alves; MOREIRA, José António Marques. Perceção de competências de aprendizagem em ambientes online no ensino da educação física. Informática na Educação: teoria e prática, Porto Alegre, v. 17, n. 1, p. 155-168, jan./jun. 2014.

\author{
Maria da Glória Oliveira Alves Ribeiro \\ Escola Básica Vilar de Andorinho \\ José António Marques Moreira \\ Universidade Aberta
}

\section{Introdução}

A educação online dinamizada por ambientes virtuais de aprendizagem, abriu novas oportunidades nos processos de ensino e aprendizagem, quer em modalidades de $b$ -learning ou e-learning.

A necessidade de (re)pensar o processo de ensino-aprendizagem tornou-se um imperativo que parece acompanhar a perceção de que a utilização das tecnologias em contexto educativo torna o ensino mais dinâmico e eficaz, mais objetivo e realista do meio que envolve o aluno e a escola, e no qual o aluno terá de (con)viver e atuar (BABIN, 1993).

O desenho e o desenvolvimento de um ambiente online que possa ser uma boa experiência pedagógica e didática implica que se envolvam os alunos e que se atenda às suas necessidades, que se otimize o tempo de 
aprendizagem e que possibilite uma prática que seja significativa. A transição de um ensino estritamente institucionalizado para uma situação de partilha colaborativa do conhecimento, sem barreiras espaciais e temporais, levanta, no entanto, algumas questões, nomeadamente da eficácia e validade desse conhecimento.

Os ambientes online e sua utilização lançam, assim, novos desafios na organização de recursos e atividades e na sua exploração de acordo com os objetivos de aprendizagem, enquanto processo que se pretende interativo e colaborativo (MOREIRA, 2012).

A forma como é experienciada a exploração do ambiente online de aprendizagem refletir-se-á na aferição da sua eficácia e na perceção que cada um terá da sua vivência em modelos de aprendizagem autónomos e virtuais. Revela-se, assim, importante aferir da perceção das competências de aprendizagem experienciadas por cada um.

Os modelos de aprendizagem implementados em ambiente online têm o atributo de alimentar a reflexão e de poderem servir como referenciais para a delineação, a implementação de práticas e a análise dos processos de interação e colaboração no seio das comunidades virtuais de aprendizagem (GARRISON; KANUCA, 2004).

No entanto, importa ter presente que a implementação de práticas de educação online deve ser acompanhada de um processo de aferição das mesmas como forma de recolha de informações úteis que permitam julgar da adequação de possíveis soluções práticas.

Deste modo, consideramos que estudos deste cariz são necessários para se poder caraterizar o impacto da implementação de ambientes online e aferir da perceção dos alunos quanto às suas competências de aprendizagem, já que constatámos não existirem estudos em Portu- gal, no âmbito do ensino fundamental, mais especificamente na área da educação física.

O estudo que agora apresentamos visa, pois, analisar o impacto de novos cenários de aprendizagem, numa modalidade em $b$-learning, nas perceções de competência de autoaprendizagem de alunos do $6^{\circ}$ ano do ensino fundamental na disciplina de educação física, nomeadamente, no que diz respeito à auto-suficiência, responsabilidade, auto-direcção e auto-regulação, confiança nas próprias competências, problematização, planificação e tomada de decisões, na aplicação de conhecimentos a situações práticas, de investimento e motivação para aprender, bem como de exploração e aprofundamento das aprendizagens, com reflexo nos resultados melhorados.

O conceito de competência de aprendizagem aqui em análise é uma variável preditiva da relação académica, relacionada com uma atitude de abertura em relação às oportunidades de aprendizagem, proporcionadas pelas experiências do dia-a-dia, e com a capacidade de utilizar de forma eficaz essas experiências formais e informais. Assim, empenhar-se na aprendizagem é despertar em si mesmo as capacidades de auto-suficiência, de auto-responsabilidade, de auto-confiança na prossecução dos objetivos e de participação ativa nos vários contextos sociais, qualidades estas necessárias em todas as situações de vida (NYHAN, 1996).

Como se sabe, a capacidade para aprender por si mesmo é, atualmente, um requisito essencial para ter sucesso em contexto escolar. Um estudante autónomo é aquele que consegue identificar uma necessidade de aprendizagem e que usa os seus recursos pessoais eficazmente, utilizando de forma sistemática e flexível as suas capacidades cognitivas, sociais e de criatividade (LIMA SANTOS et al, 2000). 
Com efeito, a capacidade de aprender por si mesmo é uma capacidade humana básica, que se converte num requisito essencial para viver no mundo atual, constituindo-se a autoaprendizagem como uma forma de vida. No entanto, deve ter-se em conta que aprender a aprender exige intencionalidade, esforço, disciplina e responsabilidade, não se confundindo com simplicidade, facilitismo ou superficialidade do processo de aprendizagem (LIMA SANTOS; GOMES, 2009).

Mais do que aprender, aprender a aprender constitui um importante meio para progredir, para enriquecimento e bem-estar pessoal e social. Esta dimensão controlada e de regulação para a promoção da capacidade de competir, cooperar e agir é, cada vez mais, determinante para o indivíduo e para a sociedade, pelos conhecimentos que soube adquirir, construir e mobilizar (LIMA SANTOS et al, 2000). Rurato (2008) corrobora a ideia de que a competência de autoaprendizagem se aplica tanto às situações de aprendizagem convencionais e formais, como às experiências informais de aprendizagem proporcionadas pelas situações do dia-a-dia. O mesmo autor salienta que os indivíduos que possuam esta competência, encaram a aprendizagem como um acontecimento natural de todos os dias e são capazes de explorar as oportunidades com a utilização eficaz das experiências de ensino formais e estruturadas, tanto quanto beneficiam dos sistemas de transmissão multimédia e de aprendizagem aberta (RURATO, 2008).

Atualmente, muitas vezes associado a uma filosofia educacional de aprendizagem centrada no estudante, o termo autoaprendizagem emerge em ambientes de aprendizagem online. Quer dizer, a relação entre autoaprendizagem e o modelo educacional proposto é ampla e flexível, permitindo várias modalidades de condução do processo, seja presencial ou a distância (ALONSO et al, 2005). A aposta na autoaprendizagem apõe em relação direta o aluno, os objetos de aprendizagem, e os conteúdos, enquanto separa o aluno no centro do processo dos agentes educacionais externos.

Empenhar-se na autoaprendizagem é despertar em si mesmo as capacidades de autossuficiência, de autorresponsabilidade, de autoconfiança na capacidade de atingir objetivos e de participação ativa nos vários contextos (LIMA SANTOS et al, 2000).

Também Magalhães (2011) refere que a autoaprendizagem deve ser definida como a competência para aprender de forma pró-ativa, responsável e autónoma, numa lógica em que é o próprio estudante que (re)constrói o seu percurso de aprendizagem, elege os conteúdos a adquirir e autorregula o processo de aprender (sem que o faça necessariamente de forma solitária).

Mais do que um processo pelo qual o estudante pode adquirir conhecimento, instruir-se e estudar de forma autónoma e baseada nos conteúdos disponibilizados, a autoaprendizagem pode permitir que os aprendentes aprendam de forma ativa, autónoma e responsável, respeitando o seu próprio ritmo de aprendizagem e desenvolvimento; aprendam por sua iniciativa, dirigindo o seu próprio processo de aprendizagem; atualizem e renovem os seus saberes e conhecimentos de acordo com as suas necessidades; construam os seus saberes e conhecimentos que Ihes vão permitir lidar com posteriores desafios, e, valorizem e complementem a sua formação (RURATO, 2008).

Assim, baseados nestes pressupostos consideramos ser fundamental investir em estratégias que promovam o sentimento de competência de aprendizagem. 
Na relação pedagógica, as orientações pelo professor-tutor serão fatores de facilitação das construções pessoais e partilhas de aprendizagem. O papel do professor é, pois, de moderação. Neste contexto, o quadro concetual que tomamos como referência é o modelo de emoderating, de Salmon (2000). Este modelo é geralmente considerado como uma das propostas mais completas e integradas, no que se refere ao papel do professor nestes ambientes educativos. O modelo desenvolvido por Gilly Salmon (2000) é baseado em cinco níveis ou etapas, que orientam a atividade do professor- moderador no trabalho com os estudantes, para conseguir a construção de comunidades virtuais de aprendizagem. Esta é uma das propostas mais estruturadas para o desenvolvimento de comunidades de aprendizagem, onde a contribuição de cada estudante, tem o seu próprio significado, e a função do professor (e-moderador) é uma função estruturante de base. É, na essência, um modelo que assenta na atividade do e-moderador e visa a independência do estudante, no trabalho com os outros elementos do grupo. Segundo Salmon (2000), para que o processo de aprendizagem online tenha êxito, os estudantes necessitam de apoio mediante um processo estruturado de desenvolvimento. Esse apoio assenta nas cinco etapas seguintes, que conduzem progressivamente, os participantes, a uma maior autonomia na aprendizagem: acesso e motivação, socialização online, troca de informação, construção de conhecimento, e desenvolvimento.

Em síntese, a nossa investigação pretende perspetivar possíveis cenários e designs alternativos de aprendizagem no ensino da educação física, em modalidades de $b$-learning, no domínio da pedagogia no ensino fundamental, estudando o efeito deste modelo na competência de aprendizagem dos estudantes.

\section{Metodologia}

Neste estudo pretendemos, fundamentalmente, e como já referido, analisar o impacto dos ambientes virtuais de aprendizagem e de um modelo pedagógico online na perceção de competência de aprendizagem de estudantes do ensino fundamental. A natureza da indagação levou-nos a considerar pertinente uma abordagem como a de Design Based Research (DBR) que parte do conceito de design experiments (WANG; HANNAFIN, 2005; RAMOS et al, 2010). De acordo com Wang e Hannafin (2005) esta metodologia de pesquisa em educação predispõe-se a realizar investigação rigorosa e reflexiva para testar e aperfeiçoar ambientes de aprendizagem inovadores. Os professores assumem o papel de co-pesquisadores, contribuindo para o desenvolvimento da teoria do design, a fim de conduzir à implementação das inovações.

Trata-se de uma metodologia que procura pesquisar problemas educativos em contextos reais de atuação pedagógica, com vista à resolução de problemas significativos e práticos, conciliando teoria e prática através de uma ligação colaborativa entre investigadores e profissionais que procuram entender, documentar, interpretar e melhorar a prática educativa.

A DBR de acordo com Wang e Hannafin (2005) está associada a uma epistemologia pragmática que considera a teoria de aprendizagem conseguida de forma colaborativa pelos intervenientes no processo, e onde o objetivo da pesquisa é resolver problemas reais, ao mesmo tempo que permite a construção de princípios de design que podem influenciar decisões futuras.

Com efeito, a DBR representa um novo paradigma de investigação no aprender a ensinar e tem-se constituído como: uma estratégia me- 
todológica sistemática e flexível que tem como objetivo melhorar as práticas dos professores através da reflexão interativa (WANG; HANNAFIN, 2005); uma estratégia de investigação inovadora que envolve a construção de uma teoria inspirada num plano que é testado em contexto natural (BARAB; ARACI; JACKSON, 2005); uma estratégia metodológica de caráter qualitativo e quantitativo que tem implicações no desenvolvimento de novas teorias de ensino e aprendizagem (DEDE, 2005); e uma estratégia que permite o desenvolvimento de ferramentas tecnológicas e de teorias que podem ser usadas para a compreensão de como os alunos aprendem (BARAB; SQUIRE, 2004).

Ao centrar-se nos processos de ensino-aprendizagem, nas propriedades do objeto/ artefacto de aprendizagem e também nos conhecimentos do professor (disciplinares, científicos, didáticos,...) em contexto real de sala de aula, a DBR permite, assim, por um lado, a aproximação entre a investigação educacional e a prática pedagógica e, por outro, a construção de conhecimento educacional a partir da prática; onde pesquisador, professores e alunos deixam de ter papéis fixos em todo o processo; e onde existe uma revisão contínua e flexível do design do projeto, tendo em vista o seu sucesso na prática existindo, por isso, momentos de redesign.

\subsection{Participantes}

No nosso estudo participaram 66 sujeitos de quatro turmas do $6^{\circ}$ ano de escolaridade do ensino fundamental, oriundos da Escola Básica Vilar de Andorinho, inserida no Agrupamento de Escolas de Oliveira do Douro n. ${ }^{\circ}$ 1, do concelho de Vila Nova de Gaia, distrito do Porto, Portugal.
Trata-se de uma amostra por conveniência, a qual se sustentou na facilidade de acessibilidade aos alunos e o cumprimento dos limites temporais definidos para a recolha de dados, ainda que, corra o risco de não ser representativa no que concerne à generalização de resultados (FORTIN et al, 2009).

Foi critério de inclusão a participação na disciplina de educação física, na unidade didática de Ginástica ao Solo disponibilizada numa plataforma de aprendizagem em regime de $b$ -learning, funcionando o ambiente online como complemento às aulas presenciais.

No estudo participaram 39 rapazes $(59,1 \%)$ e 27 raparigas $(40,9 \%)$, com idades compreendidas entre os 10 e os 14 anos.

\subsection{Instrumento}

$\mathrm{O}$ instrumento que serviu de base à recolha de dados foi a Escala de Competência de Auto-aprendizagem - ECAA (LIMA SANTOS et al, 2000) adaptada nos seus 24 itens a ambientes online, para o que os autores concederam autorização, atendendo à pertinência dos estudos de autoaprendizagem nestes "novos" ambientes.

A Escala de Competência de Auto-aprendizagem - ECAA, como referimos, é constituída por 24 itens, sendo cada um avaliado numa escala de tipo Likert de 5 pontos em que "1-Discordo Totalmente" indica uma baixa competência e "5- Concordo Totalmente" indica uma elevada competência, refletindo o grau em que cada indivíduo se auto-caracteriza em cada domínio de competência. Os itens da ECAA estão organizados em 3 dimensões gerais: (i) Aprendizagem Ativa ou Aceitação da Responsabilidade Pessoal pela Aprendizagem; (ii) Iniciativa na Aprendizagem e Orientação para a Experiência; e (iii) Autonomia na Aprendizagem. 
A primeira designada por:

(i) Aprendizagem Ativa ou Aceitação da Responsabilidade Pessoal pela Aprendizagem avaliando a perceção da capacidade para aprender em várias situações e com os outros e a aceitação da responsabilidade pessoal pelo processo de aprendizagem, formada por 12 itens: (1) "Faço perguntas, nas salas de aula virtuais (fóruns) quando tenho dúvidas"; (4) "Procuro aplicar na prática o que aprendo"; (7) Procuro todas as informações de que preciso para saber mais em ambientes online; (10) Sou uma pessoa mais atenta às participações dos outros nas salas de aula virtuais para aprender com eles; (13) "Sou capaz de aprender a ultrapassar as dificuldades que me surgem em ambientes online"; (16) "Estou sempre a aprender com as atividades desenvolvidas pelo professor no sistema de gestão de aprendizagem (plataforma)"; (19) "Aprendo sempre algo de novo com as atividades no sistema de gestão de aprendizagem (plataforma)"; (20) "Nas salas de aula virtuais (fóruns) sou capaz de aprender com pontos de vista diferentes dos meus"; (21) "Sei que sou capaz de aprender com os meus erros em ambientes de aprendizagem online"; (22) "Em ambientes de aprendizagem online sou capaz de analisar velhos problemas de novas maneiras"; (23) "Procuro aprender em todas as situações proporcionadas pelo professor no sistema de gestão de aprendizagem (plataforma)" e (24) "Gosto de aprender em ambientes online para melhorar pessoal e academicamente".

(ii) Iniciativa de Aprendizagem e Orientação para a Experiência, que avalia a orientação da aprendizagem para a experiência de problemas concretos, bem como a iniciativa na escolha das aprendizagens, formada por 6 itens: (2) "Oriento as minhas aprendizagens em ambiente online em função de problemas concretos."; (5) "Tenho em conta a minha experiên- cia quando escolho novas aprendizagens"; (8) "Sou capaz de gerir cada vez melhor as minhas aprendizagens em ambiente online"; (11) "Em ambiente online dirijo as minhas aprendizagens para o que me é útil"; (14) "Sou capaz de decidir o que devo aprender em ambiente online" e (17) "Em ambientes online sou responsável pelas minhas aprendizagens".

(iii) e Autonomia na Aprendizagem, que avalia a autonomia nas aprendizagens em função das necessidades pessoais, perfazendo 6 itens: (3) "Sou uma pessoa mais ativa nas atividades do sistema de gestão de aprendizagem (plataforma) quando sei as razões do que vou aprender"; (6) "Tenho vontade de aprender por mim mesmo em ambientes online"; (9) "A minha capacidade para aprender por mim mesmo em ambiente online está a mudar"; (12) "Em ambientes de aprendizagem online sei melhor do que as outras pessoas o que preciso de aprender"; (15) "Em ambiente online aprendo bem aquilo que melhor me permite enfrentar situações reais"; e (18) "Em ambientes online aprendo melhor aquilo que preciso para executar bem as minhas tarefas".

Desta forma, mantivemos a estrutura do conceito de autoaprendizagem, com ajuste às situações de aprendizagem em ambiente online.

\subsection{Princípios, componentes do ambiente educativo e o contexto de aplicação}

Antes de conceber o design do ambiente educativo da disciplina foi necessário ter em conta alguns princípios que podem ser generalizados para ambientes online, nomeadamente: (i) o design deve centrar-se na aprendizagem, visando atingir objetivos específicos, 
realizáveis e mensuráveis; (ii) deve centrar-se no desempenho ou realizações significativas; (iii) deve permitir que os resultados sejam medidos de uma forma fiável e válida, desenvolvendo os instrumentos necessários à avaliação do desempenho, e (iv) deve ser empírico e autorregulável.

Para além destes princípios foram, igualmente, considerados alguns componentes fundamentais para o adequado desenvolvimento do processo de ensino-aprendizagem online. Em primeiro lugar, na disciplina foi produzido um guia pedagógico de aprendizagem do aluno (GPAA) e o Plano da unidade didática de ginástica ao solo que serviu de referência para os alunos relativamente ao conteúdo, à estrutura $\mathrm{e}$ às atividades.

Procurou estabelecer-se, no seu design, uma articulação horizontal entre todos os seus elementos e uma articulação vertical inteligível. Também foi necessário descrever claramente os objetivos de aprendizagem, definidos segundo os resultados previstos, e não apenas centrados no conteúdo. O GPAA incluiu os recursos de aprendizagem que os estudantes deviam utilizar (e.g., livros e artigos que deviam ler e aqueles que deviam consultar a fim de aprofundarem os seus conhecimentos; vídeos, imagens e sítios na Internet relacionados com os temas de estudo), as e-atividades a realizar e os critérios de avaliação. Note-se que, apesar das orientações específicas do guia, a sua aplicação foi flexível. Os princípios de flexibilidade e adaptabilidade considerados indispensáveis em ambientes construtivistas por Garrison e Anderson (2005) estiveram sempre presentes.

Em segundo lugar, foram disponibilizados na plataforma diferentes recursos relacionados com os objetivos de aprendizagem. Artigos versando os temas discutidos e conteúdos multimédia online (áudio e vídeo) foram colocados à disposição dos estudantes, visando motivar e criar um vínculo entre os estudantes e o professor. Entre os recursos destacamos os nove vídeos realizados (Figuras 1 e 2), referentes a exercícios demonstrativos de elementos técnicos abordados nas aulas presenciais, com a participação de uma ginasta, da mesma idade dos alunos participantes no estudo. Foram utilizadas imagens reais, captadas na própria escola, por uma câmara de vídeo digital e posteriormente editadas no programa Magix Vídeo Deluxe 17 HD.

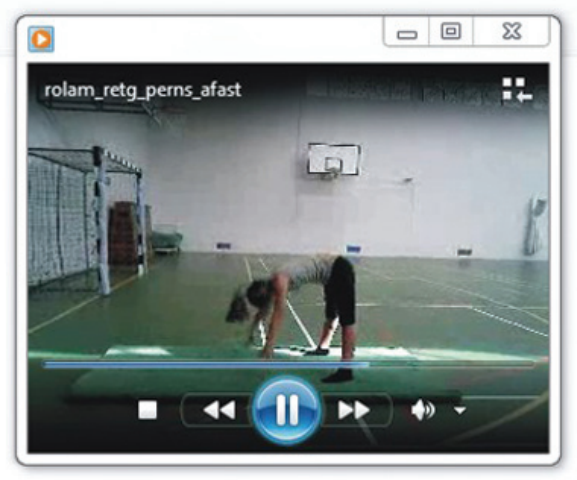

Figura 1. Rolamento à retaguarda

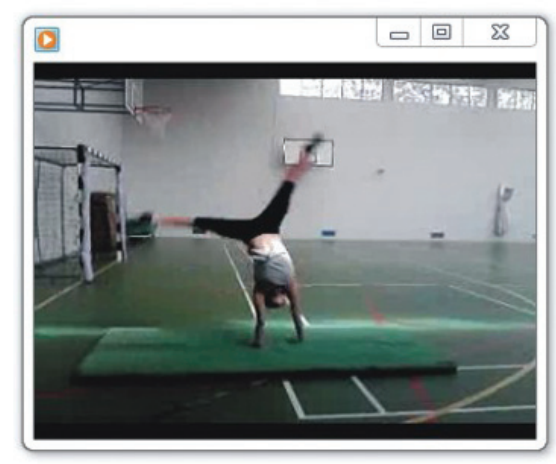

Figura 2. Roda

Em terceiro lugar, houve uma enorme preocupação com o desenvolvimento das e-atividades a realizar pelos alunos (Figura 3), ou seja, focando todo o processo nos problemas que estes deviam resolver e, consequentemente, 
desenvolvendo experiências de aprendizagem (individuais e colaborativas).

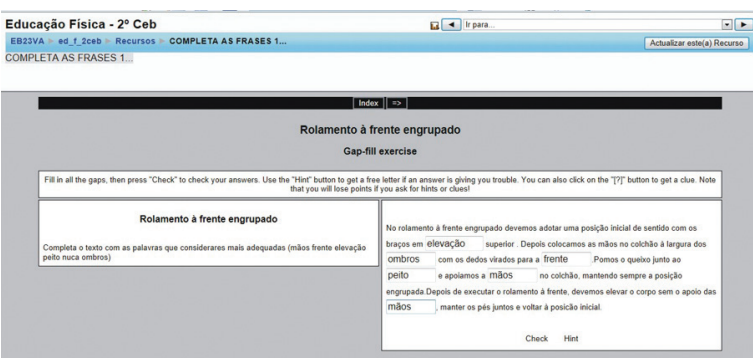

Figura 3. Recurso da ferramenta JClose Software Hotpotatoes

Em quarto lugar, a dinamização das salas de aula virtuais (fóruns), através da comunicação assíncrona, foi um elemento determinante e estruturante de todo o processo educativo. Como resultado, procurou promover-se a comunicação assíncrona nas salas de aula virtuais em todos os tópicos das unidades curriculares, através de três tipos de padrões de comunicação: (i) interação do(s) estudante(s)-conteúdo, (ii) interação do(s) estudante(s)-professor e (iii) interação do(s) estudante(s)-estudante(s). O fórum foi o meio privilegiado de comunicação para permitir a realização dos debates entre os estudantes e o professor. $\mathrm{E}$ para que efetivamente, os alunos soubessem participar e interagir de forma adequada nestes espaços foi criada uma aplicação multimédia, um protótipo intitulado "Boas práticas de participação nos fóruns" (Figuras 4 e 5), que construímos segundo o processo de ADDIA (ALLEN, 2007).

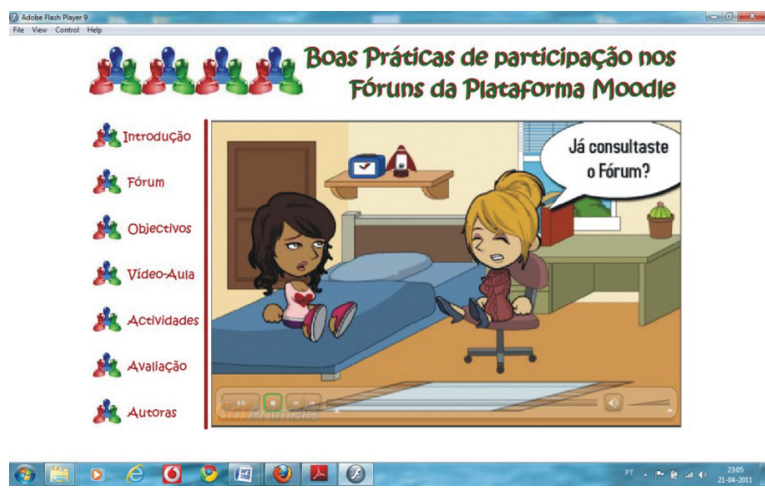

Figura 4. Versão final da interface de abertura do protótipo

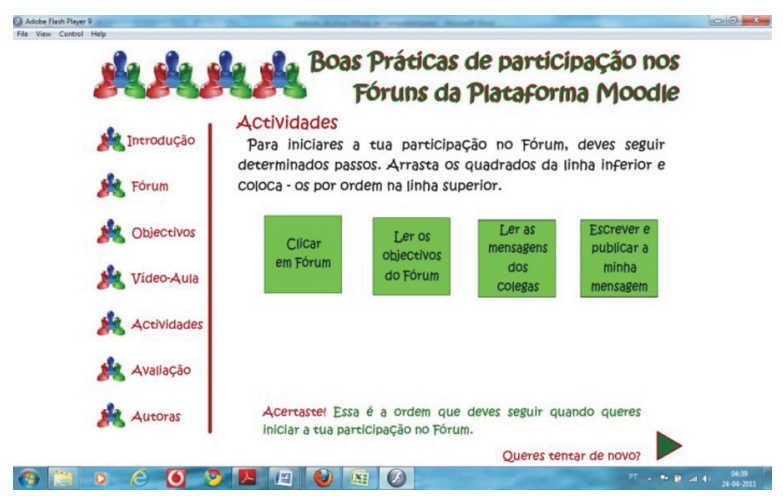

Figura 5. Porção de atividades

\section{Resultados}

Os dados foram analisados informaticamente com recurso ao software SPSS (Statistical Package For The Social Sciences - Versão 17).

Os procedimentos estatísticos adotados passaram pela estatística descritiva e inferencial. Na análise descritiva destacamos as medidas de tendência central (em particular a média), o desvio padrão como medida de dispersão, o valor mínimo e o valor máximo. Para a análise comparativa, considerámos a distribuição dos resultados e a dimensão da amostra. Não obstante a maior robustez dos 
testes paramétricos e a dimensão da amostra ( $\mathrm{N}>30)$, as distribuições não se revelaram simétricas e mesocúrticas e, por outro lado, não foram encontrados estudos prévios em ambientes online que nos permitissem pressupor que as variáveis em análise cumprissem uma distribuição normal na população em questão. Como tal, recorremos à estatística não paramétrica, utilizando o teste $U$ de Mann-Whitney (MAROCO, 2007).

Para cada sujeito, procedeu-se à recodificação das variáveis que compõem as três dimensões da escala aplicada, através da razão entre o somatório dos valores obtidos nos itens que as integram e o número de total de itens de cada subescala. Foram considerados significativos todos os resultados com intervalo de confiança superior a 95\% $(p<0.05)$.

Apresentamos os resultados do nosso estudo empírico em quadros que ilustram as frequências de resposta apresentadas pelos alunos no questionário.

Relativamente à perceção dos alunos na capacidade para aprenderem ativamente e na aceitação da responsabilidade pelas suas aprendizagens, verifica-se uma média de 4.1, indiciadora de uma perceção favorável, com uma dispersão avaliada pelo desvio padrão de 0.51, com um mínimo de 2.75 e um máximo de 5 .

\begin{tabular}{|c|c|c|}
\hline \multicolumn{3}{|c|}{$\begin{array}{c}\text { Aprendizagem ativa e aceitação da respon- } \\
\text { sabilidade pessoal pela aprendizagem }\end{array}$} \\
\hline $\mathrm{N}$ & Validados & 66 \\
\hline \multicolumn{2}{|c|}{ Média } & 4.1 \\
\hline \multicolumn{2}{|c|}{ Desvio-padrão } & .51 \\
\hline \multicolumn{2}{|c|}{ Mínimo } & 2.75 \\
\hline \multicolumn{2}{|c|}{ Máximo } & 5.00 \\
\hline
\end{tabular}

Quadro 1. Média, desvio-padrão, valor mínimo e máximo relativos à dimensão $\mathrm{F} 1$
No que diz respeito à iniciativa da aprendizagem e orientação para a experiência, a perceção dos alunos é ainda mais positiva, observando-se uma média de 4.1, indiciadora de uma perceção claramente favorável, com uma dispersão avaliada pelo desvio padrão de 0.47 , com um valor mínimo de 2.6 e um valor máximo de 5 .

\begin{tabular}{|c|c|c|}
\hline \multicolumn{3}{|c|}{$\begin{array}{c}\text { Iniciativa na aprendizagem e orientação para } \\
\text { a experiência }\end{array}$} \\
\hline $\mathrm{N}$ & Validados & 66 \\
\hline \multicolumn{2}{|c|}{ Média } & 4.10 \\
\hline \multicolumn{2}{|c|}{ Desvio-padrão } & .472 \\
\hline \multicolumn{2}{|c|}{ Mínimo } & 2.67 \\
\hline \multicolumn{2}{|c|}{ Máximo } & 5.00 \\
\hline
\end{tabular}

Quadro 2. Média, desvio-padrão, valor mínimo e máximo relativos à dimensão F2

No que diz respeito à dimensão Autonomia na Aprendizagem verifica-se uma média de 4.0, indiciadora de uma perceção favorável, com uma dispersão avaliada pelo desvio padrão de 0.45 , com um mínimo de 3.00 e um valor máximo de 4.8 .

\begin{tabular}{|c|c|c|}
\hline \multicolumn{3}{|c|}{ Autonomia na aprendizagem } \\
\hline $\mathrm{N}$ & Validados & 66 \\
\hline \multicolumn{2}{|c|}{ Média } & 4.06 \\
\hline \multicolumn{2}{|c|}{ Desvio-padrão } & .451 \\
\hline \multicolumn{2}{|c|}{ Mínimo } & 3.00 \\
\hline \multicolumn{2}{|c|}{ Máximo } & 4.83 \\
\hline
\end{tabular}

Quadro 3. Média, desvio-padrão, valor mínimo e máximo relativos à dimensão F3

Por sua vez comparando a perceção dos rapazes e das raparigas na capacidade para aprenderem ativamente ou na aceitação da responsabilidade pelas suas aprendizagens verificamos que a média obtida pelos rapazes (média 4.07) foi inferior à das raparigas (média 4.14), tal como se pode ver no quadro 4. 


\begin{tabular}{|c|c|c|c|c|c|}
\hline \multicolumn{6}{|c|}{$\begin{array}{c}\text { Aprendizagem ativa ou aceitação da respon- } \\
\text { sabilidade pessoal pela aprendizagem }\end{array}$} \\
\hline Sexo & N & Média & DP & Mínimo & Máximo \\
\hline Masculino & 39 & 4,07 & 0,53 & 2,92 & 5,00 \\
\hline Feminino & 27 & 4,14 & 0,48 & 2,75 & 4,92 \\
\hline
\end{tabular}

Quadro 4. Média, desvio-padrão, valor mínimo e máximo relativos à dimensão $\mathrm{F} 1$ em função do sexo.

No sentido de averiguarmos a significância estatística desta diferença, necessitamos de assegurar a condição de normalidade e homogeneidade das variâncias. Como se pode ver no quadro 5, através do teste de Shapiro-Wilk, os grupos não apresentam uma distribuição normal $(W=0.904, p=0.000$, no grupo dos rapazes e $\mathrm{W}=0.953, \mathrm{p}=0.016$, no grupo das raparigas).

\begin{tabular}{|c|c|c|c|c|c|c|}
\hline & \multicolumn{3}{|c|}{$\begin{array}{c}\text { Kolmogorov- } \\
\text { Smirnov }\end{array}$} & \multicolumn{3}{c|}{ Shapiro-Wilk } \\
\hline Sexo & Statistic & df & Sig. & Statistic & df & Sig. \\
\hline Masculino & .125 & 39 & .125 & .951 & 29 & .092 \\
\hline Feminino & .187 & 27 & .016 & .860 & 37 & .002 \\
\hline
\end{tabular}

Quadro 5. Teste de normalidade

Apesar da condição de homogeneidade de variâncias estar assegurada (teste de Levene com valor de significância: $p>0,05)$, aplicou-se um teste não paramétrico, designadamente ao U de Mann-Whitney, que revela não existirem diferenças significativas entre rapazes e raparigas. cujos valores se encontram nos quadros 6 e 7 .

\begin{tabular}{|l|c|c|c|c|}
\hline & $\begin{array}{c}\text { Levene } \\
\text { Statistic }\end{array}$ & df1 & df2 & Sig. \\
\hline Based on Mean & 1.259 & 1 & 64 & .266 \\
\hline Based on Median & 1.682 & 1 & 64 & .199 \\
\hline $\begin{array}{l}\text { Based on Median and } \\
\text { with adjusted df }\end{array}$ & 1.682 & 1 & 58.121 & .200 \\
\hline $\begin{array}{l}\text { Based on trimmed } \\
\text { mean }\end{array}$ & 1.463 & 1 & 64 & .231 \\
\hline
\end{tabular}

Quadro 6. Teste de homogeneidade de variâncias

\begin{tabular}{|l|r|}
\hline Mann-Whitney U & 504.500 \\
\hline Wilcoxon W & 1284.500 \\
\hline Z & -.288 \\
\hline Asymp. Sig. (2-tailed) & .773 \\
\hline
\end{tabular}

Quadro 7. Teste de Mann-Whitney

No que diz respeito à comparação entre rapazes e raparigas na segunda dimensão a média obtida pelos rapazes (média $=4.08$ ) foi, também inferior à das raparigas (média $=4.13)$.

\begin{tabular}{|c|c|c|c|c|c|}
\hline \multicolumn{7}{|c|}{ Iniciativa de aprendizagem e orientação } \\
para a experiência \\
\hline Sexo & N & Média & DP & Mínimo & Máximo \\
\hline Masculino & 39 & 4,08 & 0,43 & 3,17 & 5 \\
\hline Feminino & 27 & 4,13 & 0,52 & 2,67 & 5 \\
\hline
\end{tabular}

Quadro 8. Média, desvio-padrão, valor mínimo e máximo relativos à dimensão $\mathrm{F} 2$ em função do sexo

Da observação do quadro 8, mais uma vez verifica-se uma vantagem em termos de média do sexo feminino. No sentido, também, de averiguarmos a significância estatística desta diferença, recorremos ao teste de ShapiroWilk, o qual evidencia uma distribuição não normal relativamente à iniciativa da aprendizagem $(p>0,05)$.

\begin{tabular}{|c|c|c|c|c|c|c|}
\hline & \multicolumn{3}{|c|}{$\begin{array}{c}\text { Kolmogorov- } \\
\text { Smirnov }\end{array}$} & \multicolumn{3}{c|}{ Shapiro-Wilk } \\
\hline Sexo & Statistic & df & Sig. & Statistic & df & Sig. \\
\hline Masculino & .134 & 39 & .077 & .965 & 39 & .264 \\
\hline Feminino & .213 & 27 & .003 & .889 & 27 & .008 \\
\hline
\end{tabular}

Quadro 9. Teste de normalidade

O teste de Levene com valor de significância, p > 0,05 garante a condição de homogeneidade das variâncias, justificando o recurso à estatística não paramétrica, designadamente Teste U-Mann Whitney. 


\begin{tabular}{|l|c|c|c|c|}
\hline & $\begin{array}{c}\text { Levene } \\
\text { Statistic }\end{array}$ & df1 & df2 & Sig. \\
\hline Based on Mean & .011 & 1 & 64 & .917 \\
\hline Based on Median & .015 & 1 & 64 & .903 \\
\hline $\begin{array}{l}\text { Based on Median } \\
\text { and with adjusted df }\end{array}$ & .015 & 1 & 58.125 & .904 \\
\hline $\begin{array}{l}\text { Based on trimmed } \\
\text { mean }\end{array}$ & .000 & 1 & 64 & .997 \\
\hline
\end{tabular}

Quadro 10. Teste de homogeneidade de variâncias

Os resultados do teste de Mann-Whitney, indicado no quadro 11, não evidenciam diferenças estatisticamente significativas $(U=$ 469.5, $\mathrm{N} 1=39, \mathrm{~N} 2=27, \mathrm{p}=0.453$ ).

\begin{tabular}{|l|r|}
\hline Mann-Whitney U & 469.500 \\
\hline Wilcoxon W & 1249.500 \\
\hline Z & -.751 \\
\hline Asymp. Sig. (2-tailed) & .453 \\
\hline
\end{tabular}

Quadro 11. Teste de Mann-Whitney

Quanto ao desenvolvimento da autonomia e à construção das suas aprendizagens (F3) a comparação revela-nos que a média dos rapazes (média $=4.01$ ) foi, mais uma vez, inferior à das raparigas (média $=4.12$ ).

\begin{tabular}{|c|c|c|c|c|c|}
\hline \multicolumn{7}{|c|}{ Autonomia na aprendizagem } \\
\hline Sexo & N & Média & DP & Mínimo & Máximo \\
\hline Masculino & 39 & 4,01 & 0,45 & 3,00 & 4,83 \\
\hline Feminino & 27 & 4,12 & 0,44 & 3,00 & 4,83 \\
\hline
\end{tabular}

Quadro 12. Média, desvio-padrão, valor mínimo e máximo relativos à dimensão $\mathrm{F} 3$ em função do sexo.

No sentido de analisar a significância estatística desta diferença procedemos à análise da condição de normalidade e homogeneidade das variâncias. Como se pode ver através do teste de Shapiro-Wilk, os dois grupos apresentam distribuições normais ( $p>0,05)$.

\begin{tabular}{|c|c|c|c|c|c|c|}
\hline & \multicolumn{3}{|c|}{$\begin{array}{c}\text { Kolmogorov- } \\
\text { Smirnov }\end{array}$} & \multicolumn{3}{c|}{ Shapiro-Wilk } \\
\hline Sexo & Statistic & df & Sig. & Statistic & df & Sig. \\
\hline Masculino & .113 & 39 & .200 & .974 & 39 & .487 \\
\hline Feminino & .127 & 27 & .200 & .939 & 27 & .118 \\
\hline
\end{tabular}

Quadro 13. Teste de normalidade

A condição de homogeneidade das variâncias está assegurada pelo teste de Levene ( $p$ $>0.05)$.

\begin{tabular}{|l|c|c|c|c|}
\hline & $\begin{array}{c}\text { Levene } \\
\text { Statistic }\end{array}$ & df1 & df2 & Sig. \\
\hline Based on Mean & .181 & 1 & 64 & .672 \\
\hline Based on Median & .246 & 1 & 64 & .621 \\
\hline $\begin{array}{l}\text { Based on Median } \\
\text { and with adjusted df }\end{array}$ & .246 & 1 & 63.275 & .621 \\
\hline $\begin{array}{l}\text { Based on trimmed } \\
\text { mean }\end{array}$ & .244 & 1 & 64 & .623 \\
\hline
\end{tabular}

Quadro 14. Teste de homogeneidade de variâncias

Garantidas as duas condições, recorrremos à estatística paramétrica, designadamente ao t-test para amostras independentes. Pese embora a média das raparigas (4.12) seja superior à dos rapazes (4.01), essas diferenças, avaliadas pelo t-test (-0.995), não são estatisticamente significativas $(p=0.324)$.

\section{Considerações finais}

Conforme temos vindo a afirmar (MONTEIRO; MOREIRA, 2012) o sucesso da educação em ambientes online depende não só das condições tecnológicas e sociais, mas também, e fundamentalmente, das condições pedagógicas. Estes novos ambientes obrigam a repensar constantemente os papéis dos professores e dos estudantes e a relação existente entre eles e exigem também uma nova forma de co- 
municar, onde ambos partilham a responsabilidade pela aprendizagem.

Sendo a perceção das competências de aprendizagem um indicador da eficácia das aprendizagens, o que valida, em certa medida, os modelos pedagógicos subjacentes ao processo de relação pedagógica, este estudo na área disciplinar da educação física revela que, de um modo geral, os alunos que experienciam a aprendizagem em ambientes online apresentam uma perceção favorável na capacidade para aprenderem ativamente.

Por outras palavras, podemos afirmar que os resultados do estudo mostram que o ambiente online construído, ancorado num design centrado no desenvolvimento de competências e num modelo pedagógico -e-moderating- baseado nos princípios do construtivismo, da autonomia e da interação tiveram efeitos muito positivos na perceção das competências de aprendizagem dos estudantes, nas dimensões consideradas: Aprendizagem Ativa ou Aceitação da Responsabilidade Pessoal pela Aprendizagem, Iniciativa de Aprendizagem e Orientação para a Experiência e Autonomia na Aprendizagem.

Relativamente à perceção que rapazes e raparigas têm, relativamente ao seu desempe- nho no ambiente online, importa salientar que, apesar de não existirem diferenças significativas entre ambos, os valores médios das raparigas são superiores aos valores dos rapazes nas diferentes dimensões consideradas. Em ambientes presenciais alguns estudos recentes revelam-nos que as raparigas, e fruto da sua maior maturidade, apresentam nesta fase um desempenho académico superior aos rapazes (CARVALHO, 2001; BARRIGAS; FRAGOSO, 2012), por isso não é surpresa que esta situação seja idêntica em ambientes online.

No entanto, temos que ler estes resultados com algumas reservas, já que este trabalho restringiu-se a uma escola do ensino fundamental em Portugal. Recomenda-se, pois, a realização de mais estudos nesta área com um número maior de participantes, incluindo escolas, do ensino público e particular, noutras cidades e abrangendo também zonas rurais, de modo a cobrir um maior número de alunos, níveis e instituições de ensino.

Esperamos, pois que este estudo seja um instrumento útil para aqueles que se interessam por estas temáticas e que seja um primeiro passo para outros trabalhos a realizar na área da educação física.

\section{Referências}

ALLEN, M. Designing Successful e-Learning: Forget what you knowabout instructional design and do something interesting. San Francisco: Pfeiffer, 2007.

ALONSO, F. et al. An instructional model for web-based e-learning education with a blended learning process approach. British Journl of Educational Technology, v. 36, n. 2, p. 217-235, 2005.

BABIN, P. Linguagem e Cultura dos Média. Lisboa: Bertrand Editora, 1993. 
BARAB, S.; SQUIRE, K. Design-based research: Putting a stake in the ground. The Journal of the Learning Sciences, v. 13, n. 1, p. 1-14, 2004.

BARAB, S.; ARICI, A.; JACKSON, C. Eat your vegetables and do your homework: A design-based investigation of enjoyment and meaning in learning. Educational Technology, v. 45, n. 1, p. 15-21, 2005.

BARRIGAS, C.; FRAGOSO, I. Maturidade, desempenho académico, capacidade de raciocínio e estatuto socioeconómico em crianças de Lisboa entre os 6 e os 13 anos de idade. Revista Portuguesa de Educação, v. 25, n. 1, p. 193-215, 2012.

CARVALHO, M. Mau aluno, boa aluna? Como as professoras avaliam meninos e meninas. Estudos Feministas, v. 9, n. 2, p. 554-574, 2001.

DEDE, C. Commentary: The growing utilization of design-based research. Contemporary Issues In Technology and Teacher Education, v. 5, n. 3/4, p. 345-348, 2005.

FORTIN, M. et al. Fundamentos e Etapas do Processo de Investigação. Loures: Lusodidacta, 2009.

GARRISON, D.; ANDERSON, T. El e-learning en el siglo XXI. Investigación e práctica. Barcelona: Octaedro, 2005.

GARRISON, D.; KANUKA. Blended Learning: Uncovering its Transformative Potential in Higher Education. The Internet and Higher Education, v. 7, n. 2, p. 95-105, 2004.

LIMA SANTOS, N. et al. Educação e aprendizagem de adultos: Avaliação do autoconceito de competência cognitiva e da autoaprendizagem. Revista Galego-Portuguesa de Psicoloxía e Educación, v. 6, n. 4, p. 649-656, 2000.

LIMA SANTOS, N.; GOMES, I. Transformações e tendências do ensino-aprendizagem na era do digital: alguns passos para uma arqueologia de um novo saber-poder. Revista antropológicas, n. 11, p. 143159, 2009.

MAGALHÃES, M. Autoconceito de competência e autoaprendizagem em alunos do ensino secundário: comparação de cursos científico-humanísticos com cursos profissionais. Dissertação (Mestrado). Universidade Fernando Pessoa, 2011, Porto, PT.

MARÔCO, J. Análise estatística com utilização do SPSS. Lisboa: Edições Sílabo, 2007.

MONTEIRO, A.; MOREIRA, J. A. O Blended Learning e a Integração de Sujeitos, Tecnologias, Modelos e Estratégias de Ensino-aprendizagem. In: MONTEIRO, A.; Moreira, J. A.; ALMEIDA, A. C.; LENCASTRE, J. 
A. Blended Learning em Contexto Educativo: Perspetivas teóricas e práticas de investigação. Santo Tirso: De Facto Editores, 2012, p. 33-58.

MOREIRA, J. A. Novos cenários e modelos de aprendizagem construtivistas em plataformas digitais. In: MONTEIRO, A.; MOREIRA, J.A.; ALMEIDA, A.C., Educação Online: Pedagogia e Aprendizagem em Plataformas Digitais. Santo Tirso: De Facto Editores, 2012, p. 29-46.

NYHAN, B. Desenvolver a capacidade de aprendizagem das pessoas: Perspectivas europeias sobre a competência de auto-aprendizagem e mudança tecnológica. Caldas da Rainha: Eurotecnet, 1996.

RAMOS, P. et al. A Pesquisa Baseada em Design em Artigos Científicos Sobre o Uso de Ambientes de Aprendizagem Mediados Pelas Tecnologias da Informação e da Comunicação no Ensino de Ciências. Revista de Educação em Ciência e Tecnologia, v. 3, n. 1, p.77-102, 2010.

RURATO, P. As características dos aprendentes na educação a distância - impacto no processo educativo com vista ao desenvolvimento de estratégias de sucesso. Tese (Doutoramento). Universidade de Aveiro, 2008, Aveiro, PT.

SALMON, G. E-moderating: the key to teaching and learning online. London: Kogan Page Limited, 2000.

WANG, F.; HANNAFIN, M. J. Design-based research and technology-enhanced learning environments. Educational Technology Research and Development, v. 53, n. 4, p. 5-23, 2005.

Submetido para avaliação em 23 de abril de 2013.

Aprovado para publicação em 07 de novembro de 2013.

Maria da Glória Oliveira Alves Ribeiro: Escola Básica Vilar de Andorinho - Vila Nova de Gaia - Portugal. E-mail: mgloriaribeiro@gmail.com

José António Marques Moreira: Universidade Aberta - Lisboa - Portugal. E-mail: jmoreira@uab.pt 